\title{
Prediction of Hepatocellular Carcinoma Incidence Risk by Ultrasound Elastography
}

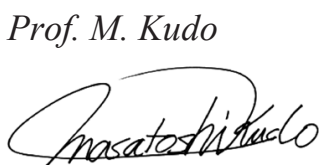

Editor Liver Cancer

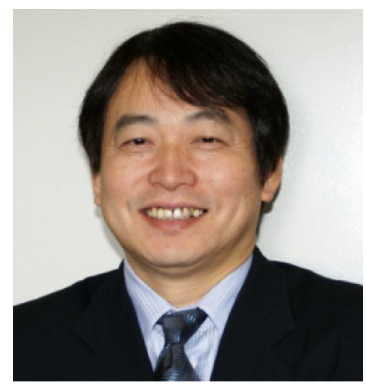

In patients with chronic hepatitis and continuous inflammation of the liver, fibrosis develops during the course of wound healing, and with recurrent accumulation of scar tissue and regenerative nodular formation, chronic hepatitis progresses to cirrhosis. In untreated patients with chronic hepatitis $C$ infection, which is a leading cause of hepatocellular carcinoma (HCC), the Metavir liver fibrosis stage (F0, no fibrosis; F4, cirrhosis)[1] increases at an annual rate of F0.1 [2], with a concurrent increase in the incidence of HCC [3]. Therefore, it is extremely important to evaluate the liver fibrosis stage during follow up of chronic hepatitis $\mathrm{C}$ so as to understand the HCC risk. However, liver biopsy, the gold standard for diagnosing liver fibrosis, is susceptible to sampling variability [4] and diagnostic variability among pathologists. In addition, repeat biopsy is not recommended because of its invasiveness, the associated pain and risk of hemorrhage. Although serum fibrosis marker and hematologybased prediction algorithms have been widely reported for evaluating liver fibrosis, the results obtained with these methods may be affected by fibrotic conditions in other organs.

Ultrasound elastography emerged as an imaging modality around 2010 and has been found in many studies to be diagnostically useful for liver fibrosis. The word "elastography" ("elasticity" + "graphy"), a collective term for the graphical display of tissue elasticity, was first used in 1991 to describe the technique of strain elastography proposed by Dr. Ophir at the University of Texas. Another term, "tissue elasticity imaging," is used to define the graphic technology used to capture tissue elasticity over a wider area. However, the terms "elastography" and "tissue elasticity imaging" are now often used interchangeably and are even applied to non-graphic quantitative measurement methods such as that employed by FibroScan.

Ultrasound elastography is classified into two major types based on the measurement principles adopted: strain elastography, which displays differences in tissue strain and is used in real-time tissue elastography (RTE), which is the first commercially available machine in the world launched in 2003 and shear wave elastography, which measures the speed of propagating waves and is used in FibroScan and Virtual Touch tissue quantification (VTQ) [5](table 1). While strain elastography enables the measurement (display) of liver fibrosis, 
Table 1. Classification of various elastography methods. Reproduced with permission from Shiina T et al.[5].

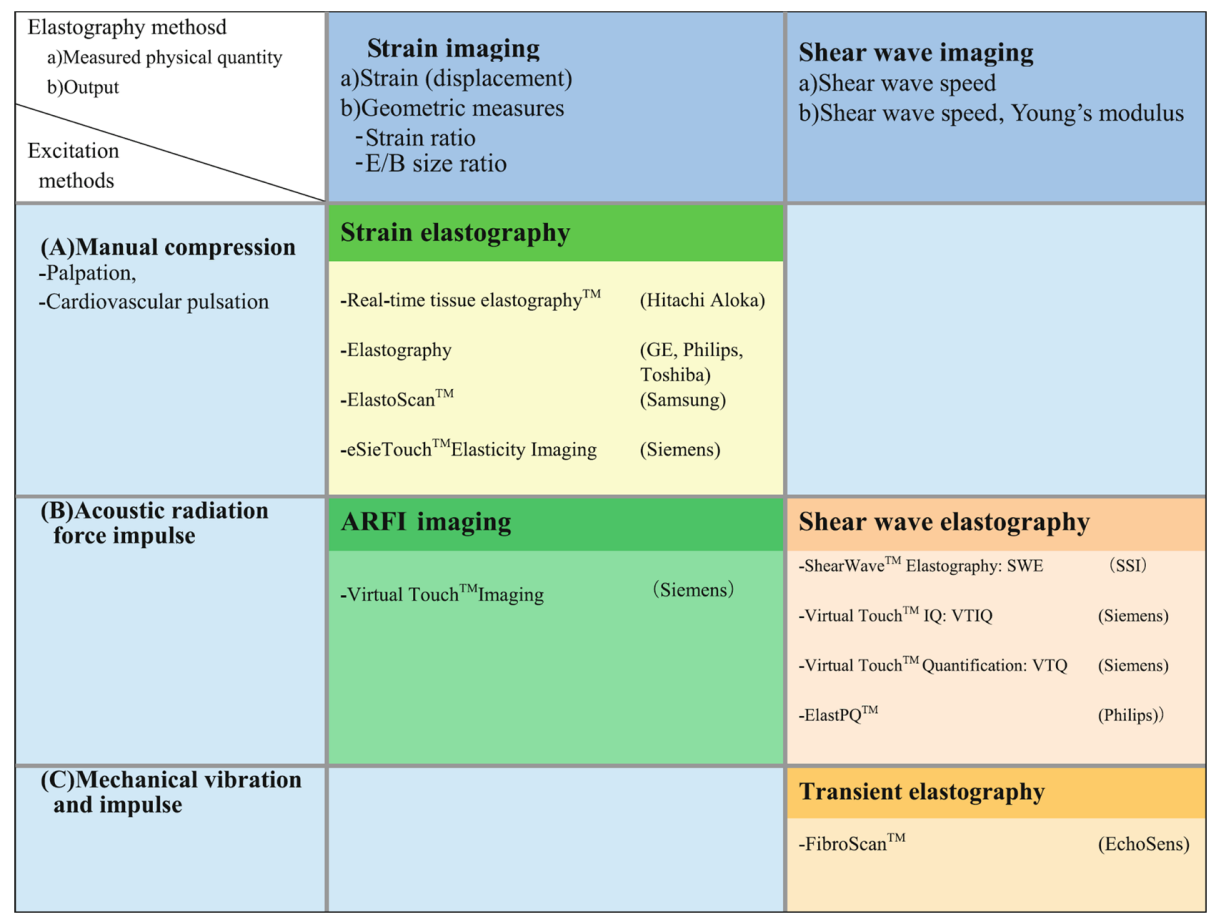

$\mathrm{E} / \mathrm{B}$ size ratio=the ratio of the size of a lesion in a stain image to its size in a B-mode image; ARFI=acoustic radiation force impulse.

measurement values in shear wave elastography are affected by inflammation, jaundice, and congestion in addition to fibrosis [6-9]. In other words, measurement values with the latter technique increase as fibrosis, inflammation, jaundice, and congestion exacerbate, and vice versa, presumably because these pathological conditions alter the density, and thereby the elasticity and viscosity, of liver tissue [10].

In 2003, the French company Echosens launched FibroScan. This device calculates the degree of liver stiffness (in $\mathrm{kPa}$ ) by sending acoustic push pulses from the body surface to the liver and following the waves by ultrasound to measure the shear wave speed. The propagation velocity of shear waves correlates with tissue stiffness, with waves traveling faster in harder tissues. FibroScan has several advantages: (1) it is a non-invasive and painless technique, (2) it provides immediate results (within $30 \mathrm{~s}$ of measurement), (3) the results are highly reproducible, (4) the measurement area is as wide as $\sim 1 / 500$ th of the total liver mass (the size of a biopsy specimen is $\sim 1 / 50,000$ th of the total liver mass), and (5) it can be safely repeated for follow up. On the down side, measurement reproducibility and even the measurement itself can be adversely affected by ascites (push pulses do not travel through fluids) and by thick layers of subcutaneous fat, narrow intercostal spaces, and severe liver atrophy.

In a meta-analysis of studies assessing the stage of liver fibrosis using FibroScan, Friedrich-Rust et al. found that the cut-off level for a diagnosis of F2 or higher was $7.65 \mathrm{kPa}$ [area under receiver operating characteristic (AUROC) curve, 0.84] and for a diagnosis of F4, the cut-off level was $13.01 \mathrm{kPa}$ (AUROC, 0.94) [11], demonstrating that fibrosis staging by FibroScan has high accuracy. However, the stages of fibrosis diagnosed based on liver stiffness do not always match the pathological stage established by liver biopsy. In a study conducted 


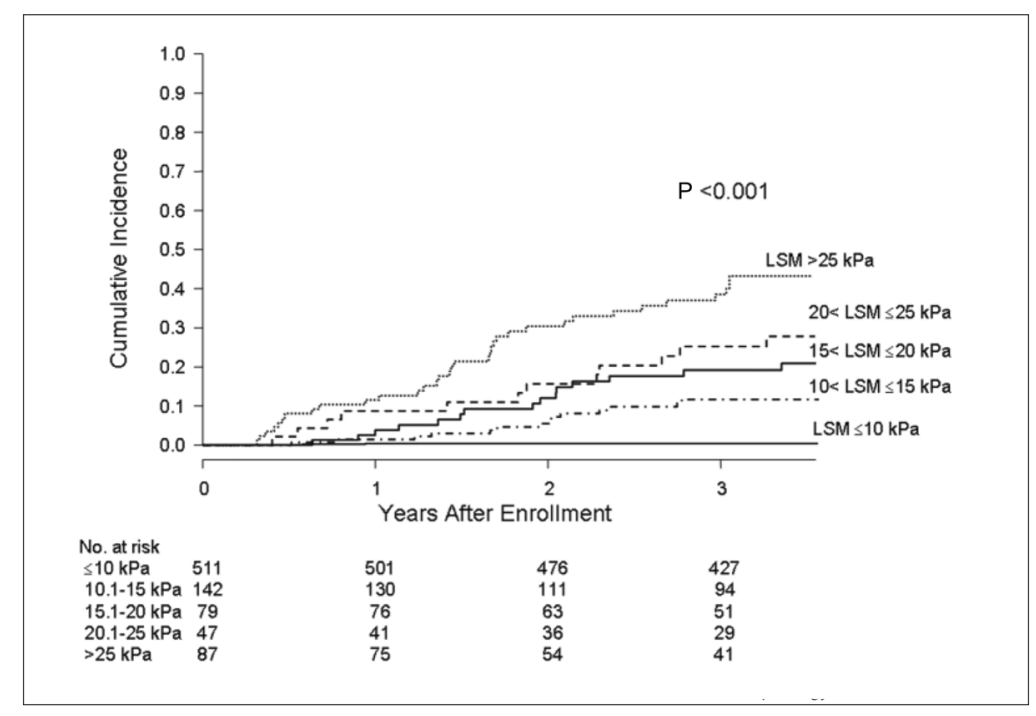

Fig. 1. Cumulative incidence of HCC development stratified based on liver stiffness measurement (LSM, $\mathrm{n}=866$ ) in patients with hepatitis C. Reproduced with permission from Masuzaki R et al.[13].

by Ichino et al., 17 (40\%) of 42 patients with F2 fibrosis determined by liver biopsy were diagnosed with F0-F1 fibrosis by FibroScan, even though an AUROC of 0.88 was taken to be F2 or higher liver fibrosis, with $81 \%$ sensitivity and $80 \%$ specificity [12].

Many studies have reported staging differences in liver fibrosis between liver stiffnessbased diagnoses and biopsy findings. During the acute phase of acute hepatitis, the liver becomes as stiff as cirrhotic liver, although the stiffness returns to normal as hepatitis improves. Moreover, even among patients with the same fibrosis stage, those with high alanine aminotransferase (ALT) levels resulting from hepatitis B virus (HBV)- or HCV-induced chronic hepatitis have a high degree of liver stiffness compared with patients with normal ALT levels owing to antiviral treatment or the natural course. This suggests that liver stiffness is affected by the severity of inflammation as well as by liver fibrosis, leading to an overestimation of liver stiffness when inflammation is present. Therefore, when staging liver fibrosis based on liver stiffness in patients with high ALT levels, it is important to keep in mind that the actual fibrosis stage might be lower. Note that strain elastography, however, is not affected by inflammation in the liver.

In a study by Masuzaki et al. investigating the relationship between cirrhosis and the incidence of HCC in 866 patients with hepatitis C, HCC developed in 77 patients over the 3-year follow-up period [13]. When patients were classified based on liver stiffness at the initial FibroScan examination, patients with a high degree of liver stiffness subsequently had a high incidence of HCC (fig. 1), and on multivariate analysis, in addition to age, sex, and albumin level, liver stiffness was an independent risk factor associated with HCC incidence [13]. Similarly, in a study by Jung et al. investigating the association between liver stiffness and the incidence of HCC in patients with hepatitis B [14], HCC developed in 57 of 1130 patients after a median follow up of 31 months (fig. 2). Multivariate analysis extracted liver stiffness at initial examination, together with age, sex, alcohol consumption, albumin level, and HB e-antigen positivity, as risk factors associated with HCC incidence.

Because liver stiffness is correlated with the stage of liver fibrosis, it is reasonable to assume that liver with a high degree of stiffness indicates advanced liver fibrosis. This strong correlation between liver stiffness and HCC risk also applies to cirrhotic patients, who have 


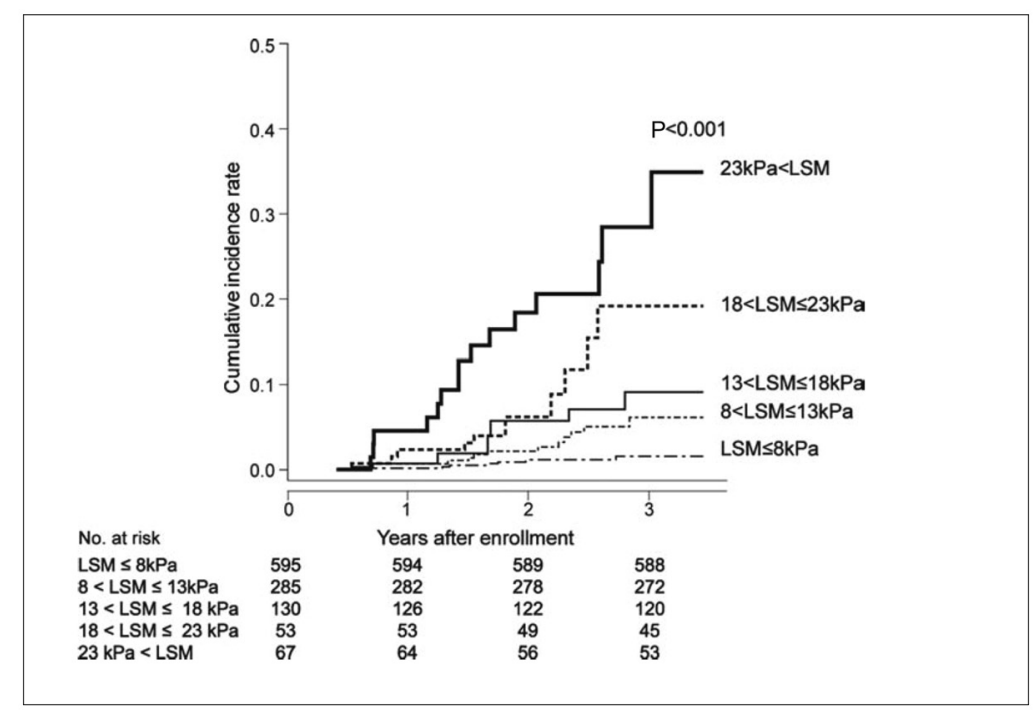

Fig. 2. Cumulative incidence rates of HCC based on stratified LSM (Kaplan-Meier plot) in patients with hepatitis B. The cumulative incidence rates increased significantly in association with higher LSM (log-rank test, $\mathrm{p}<0.0001, \mathrm{n}=1130$ ). Reproduced with permission from Jung KS et al.[14].

a high degree of liver stiffness, suggesting that liver stiffness is a useful clinical indicator to classify patients at high risk of HCC.

In summary, recent advances in ultrasound elastographic technology allow us to predict the occurrence of HCC more accurately. Conventionally, serum fibrosis markers, platelet count, and imaging findings are used to diagnose cirrhosis and the potential risk for HCC. In contrast, ultrasound elastography is a simple, non-invasive, and highly accurate method for predicting the occurrence of HCC. We recommend the proactive use of not only FibroScan, but also VTQ and RTE (table 1), in clinical settings because they are all useful in predicting the risk of a patient developing HCC.

\section{References}

1 Bedossa P, Poynard T for the METAVIR cooperative study group: An algorithm for the grading of activity in chronic hepatitis C. The METAVIR Cooperative Study Group. Hepatology 1996;24:289-293.

-2 Shiratori Y, Imazeki F, Moriyama M, Yano M, Arakawa Y, Yokosuka O, Kuroki T, Nishiguchi S, Sata M, Yamada G, Fujiyama S, Yoshida H, Omata M: Histologic improvement of fibrosis in patients with hepatitis C who have sustained response to interferon therapy. Ann Intern Med 2000;132:517-524.

-3 Yoshida H, Shiratori Y, Moriyama M, Arakawa Y, Ide T, Sata M, Inoue O, Yano M, Tanaka M, Fujiyama S, Nishiguchi S, Kuroki T, Imazeki F, Yokosuka O, Kinoyama S, Yamada G, Omata M: Interferon therapy reduces the risk for hepatocellular carcinoma: national surveillance program of cirrhotic and noncirrhotic patients with chronic hepatitis C in Japan. IHIT Study Group. Inhibition of Hepatocarcinogenesis by Interferon Therapy. Ann Intern Med 1999;131:174-181.

4 Bedossa P, Dargère D, Paradis V: Sampling variability of liver fibrosis in chronic hepatitis C. Hepatology 2003;38:1449-1457.

5 Shiina T: JSUM ultrasound elastography practice guidelines: basics and terminology. J Med Ultrasonics 2013;40:309-323.

-6 Arena U, Vizzutti F, Corti G, Ambu S, Stasi C, Bresci S, Moscarella S, Boddi V, Petrarca A, Laffi G, Marra F, Pinzani M: Acute viral hepatitis increases liver stiffness values measured by transient elastography. Hepatology 2008;47:380-384.

7 Millonig G, Reimann FM, Friedrich S, Fonouni H, Mehrabi A, Büchler MW, Seitz HK, Mueller S: Extrahepatic cholestasis increases liver stiffness (FibroScan) irrespective of fibrosis. Hepatology 2008;48:1718-1723. 
8 Sagir A, Erhardt A, Schmitt M, Häussinger D: Transient elastography is unreliable for detection of cirrhosis in patients with acute liver damage. Hepatology 2008;47:592-595.

-9 Colli A, Pozzoni P, Berzuini A, Gerosa A, Canovi C, Molteni EE, Barbarini M, Bonino F, Prati D: Decompensated chronic heart failure: increased liver stiffness measured by means of transient elastography. Radiology 2010;257:872-878.

10 Kudo M, Shiina T, Moriyasu F, Iijima H, Tateishi R, Yada N, et al: JSUM ultrasound elastography practice guidelines: liver. J Med Ultrasonics 2013;40:325-357.

-11 Friedrich-Rust M, Ong MF, Martens S, Sarrazin C, Bojunga J, Zeuzem S, Herrmann E: Performance of transient elastography for the staging of liver fibrosis: a meta-analysis. Gastroenterology 2008;134:960-974.

12 Ichino N, Osakabe K, Nishikawa T, Sugiyama H, Kato M, Kitahara S, Hashimoto S, Kawabe N, Harata M, Nitta Y, Murao M, Nakano T, Arima Y, Shimazaki H, Suzuki K, Yoshioka K: A new index for non-invasive assessment of liver fibrosis. World J Gastroenterol 2010;16:4809-4816.

13 Masuzaki R, Tateishi R, Yoshida H, Goto E, Sato T, Ohki T, Imamura J, Goto T, Kanai F, Kato N, Ikeda H, Shiina S, Kawabe T, Omata M: Prospective risk assessment for hepatocellular carcinoma development in patients with chronic hepatitis C by transient elastography. Hepatology 2009;49:1954-1961.

14 Jung KS, Kim SU, Ahn SH, Park YN, Kim do Y, Park JY, Chon CY, Choi EH, Han KH: Risk assessment of hepatitis B virus-related hepatocellular carcinoma development using liver stiffness measurement (FibroScan). Hepatology 2011;53:885-894. 